\title{
Segmentation and surface reconstruction of a cadaver heart on Mimics software
}

\author{
D.S. Shin ${ }^{1}$, S. Lee ${ }^{2}$, H.S. Park², S.-B. Lee ${ }^{2}$, M.S. Chung ${ }^{3}$ \\ ${ }^{1}$ Department of Orthopaedic Biomaterial Science, Osaka University Graduate School of Medicine, Osaka, Japan \\ ${ }^{2}$ Korea Institute of Science and Technology Information, Daejeon, Korea \\ ${ }^{3}$ Department of Anatomy, Ajou University School of Medicine, Suwon, Korea
}

[Received 8 May 2014; Accepted 19 June 2014]

The Visible Korean research team used Mimics software (Materialise, Leuven, Belgium) for the segmentation and subsequent surface reconstruction of heart structures using information obtained from sectioned images of a cadaver. Twenty-six heart components were outlined in advance on Photoshop (Adobe Systems, San Jose, CA, USA). By use of the Mimics, the outlined images were then browsed along with the vertical planes as well as the 3-dimensional surface models, which were immediately built by piling the images. Erroneous delineation was readily detected and revised until satisfactory heart models were acquired. The surface models and the selected sectioned images in horizontal, coronal, and sagittal planes were inputted into a PDF file, where any combinations of reconstructed constituents could be displayed and rotated by the user. Mimics software accelerated the segmentation and surface reconstruction of heart anatomical structures. Similar benefits hopefully result from various serial images of other organs. The PDF file, and plane and stereoscopic image data are being distributed to others, and should prove valuable for medical students and clinicians. (Folia Morphol 2015; 74, 3: 372-377)

Key words: heart, visible human projects, computer-assisted image processing, three-dimensional imaging, user-computer interface

\section{INTRODUCTION}

The Visible Korean is one of the Visible Human Projects that produce serially sectioned images of cadavers. Those are high resolution images with genuine body colour, enabling segmentation and 3-dimensional (3D) reconstruction of the fine structures. Unlike other project groups in the United States and China, the Visible Korean team has distributed the processed data as well as the raw images. Portable document format (PDF) files, composed of 3D surface models, are obtainable on the Visible Korean homepage (anatomy. co.kr). The objects of the distributed PDF files used to be the whole body of a male cadaver [8], the head and ear of another male cadaver $[4,10]$, and the pelvis of a female cadaver [9]. The next planned addition to this data library was the heart. Detailed heart compositions were already outlined on the sectioned images of new female cadaver's entire body. Delineation was performed on the Photoshop CS6 version 10 (Adobe Systems, Inc., San Jose, CA, USA), as previously described [5, 12].

Not long ago, we encountered Mimics version 17 (Materialise, Leuven, Belgium), originated to manage clinical images on the personal computer. Mimics simultaneously deals with $2 \mathrm{D}$ raw data and 3D reconstructed models. The integrating software brings about the different paradigm from our previous trials to employ a couple of software packages for the segmentation and 3D reconstruction. The software capability might also facilitate revision of the outlined images [10]. 
Table 1. Structures of the heart and adjacent locations that were outlined and surface reconstructed

\begin{tabular}{ll}
\hline Groups (no. of the structures) & Structures \\
\hline Bones (2) & Sternum, ribs \\
Pericardium (1) & Pericardium \\
Right atrium, etc. (4) & Right atrium*, superior vena cava, brachiocephalic vein, inferior vena cava \\
Right ventricle, etc. (5) & Right ventricle*, tricuspid valve, pulmonary valve, pulmonary trunk, pulmonary arteries \\
Left atrium, etc. (2) & Left atrium*, pulmonary vein \\
Left ventricle, etc. (5) & Left ventricle*, mitral valve, aortic valve, ascending aorta, arch of aorta \\
Coronary arteries (4) & Right coronary artery, left coronary artery, circumflex artery, anterior interventricular branch \\
Cardiac veins (3) & Coronary sinus, great cardiac vein, middle cardiac vein \\
\hline
\end{tabular}

${ }^{*}$ Four heart chambers involve the lumen, endocardium, myocardium, but not the pericardium.

One objective of this research was to verify whether the outlining and surface reconstruction of anatomical structures could be enhanced using Mimics software. In keeping with the grounding philosophy of the Visible Korean project, we also aimed to distribute the acquired PDF file of surface models of the heart, for use by medical students and clinicians.

\section{MATERIALS AND METHODS}

Horizontal sectioned images $(0.2 \mathrm{~mm}$ intervals; $5,616 \times 2,300$ resolution; 48 bit colour depth) were acquired from a whole female cadaver (26-years-ofage at death; $1.65 \mathrm{~m}$ length including plantar flexed foot; $52 \mathrm{~kg}$ weight) in 2010. The 615 sectioned images containing heart and adjacent great blood vessels were sorted. The representative sectioned images, which would be source of a PDF file, were chosen at $5 \mathrm{~mm}$ intervals. Using self-developed software, the raw sectioned images of heart were compiled to generate the coronal and sagittal images at $5 \mathrm{~mm}$ intervals.

It was intended to delineate 26 components of the heart, adjacent blood vessels, and front bones that were categorised into 8 groups. Great vessels were included in the heart chamber groups. However, small coronary arteries and cardiac veins were made independent. This grouping was maintained in the resulting PDF file (Table 1).

Delineation intervals were $1 \mathrm{~mm}$, not $0.2 \mathrm{~mm}$, so that every 5 sectioned images were selected for the remaining 123 images. The images were opened in Photoshop as Photoshop document (PSD) files. Structure outlines were drawn and filled with colours as described previously [5, 12]. Only colour-filled outlines were remained excluding the sectioned images, which were saved as 123 tagged image file format (TIFF) files. The TIFF files occupied more than 9 gigabytes, which was too large to place in a Mimics (MCS) file. To reduce the file size, the high resolution $(5,616 \times 2,300)$ was lessened by half to $2,808 \times 1,150$. Twenty-six outlined structures were divided into 3 bundles, according to which, colour-filled outlines were shared by 3 MCS files.

Mimics utilised brightness to recognise and cluster the serial outlines of every structure. After stacking the categorised outlines, surface reconstruction of entire structures was simultaneously achieved. The concurrently built surface models were automatically divided into a number of independent models (Fig. 1).

Faulty outlines of a heart component gave rise to unexpected steps in the coronal and sagittal planes; the outlines also produced a surface model that did not correspond with anatomical knowledge (Fig. 1). The outlines were directly revised using the Mimics manual modification tool. Anatomists modified the outlines until the secondary images were satisfactory.

Whole surface models of heart in the MCS file were saved as stereolithography files. The surface models and auxiliary 3-directional sectioned images were transferred to a PDF file. We employed previously formulated and detailed transferring methods [8]. The sole notable item was that prior to inputting, the outline colours of bones and pericardium were made semitransparent to show their interiors (Table 1). Later, the colours of surface models, already decided by developers, could be made semitransparent on Adobe Reader version 9 (Adobe Systems).

\section{RESULTS}

The outcome models were satisfactory to exhibit the comprehensive heart shape (Fig. 2). The PDF file of heart (size $45 \mathrm{MB}$ ) was obtained from the Visible Korean site (anatomy.co.kr). Our policy is availability of the information through free downloading with no required registration. Using the PDF file on Adobe Reader, any combination of the 26 surface models and 73 sectioned 


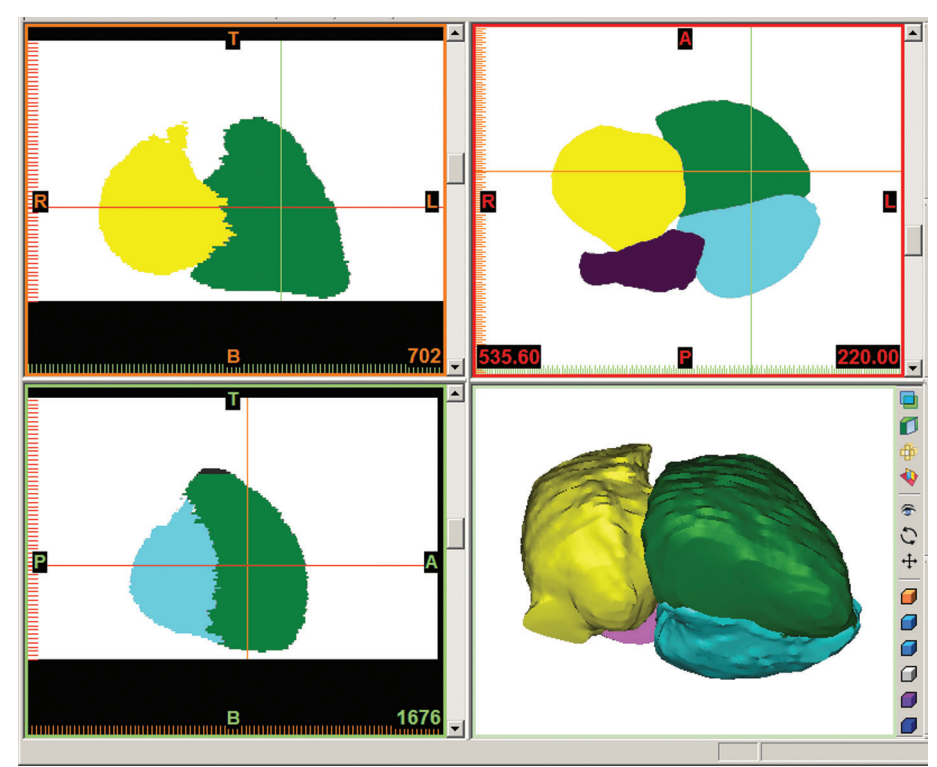

Figure 1. Mimics file visualising the original colour-filled outlines (top right), accompanied by the coronal plane (top left), sagittal plane (bottom left), and stereoscopic surface models (bottom right).

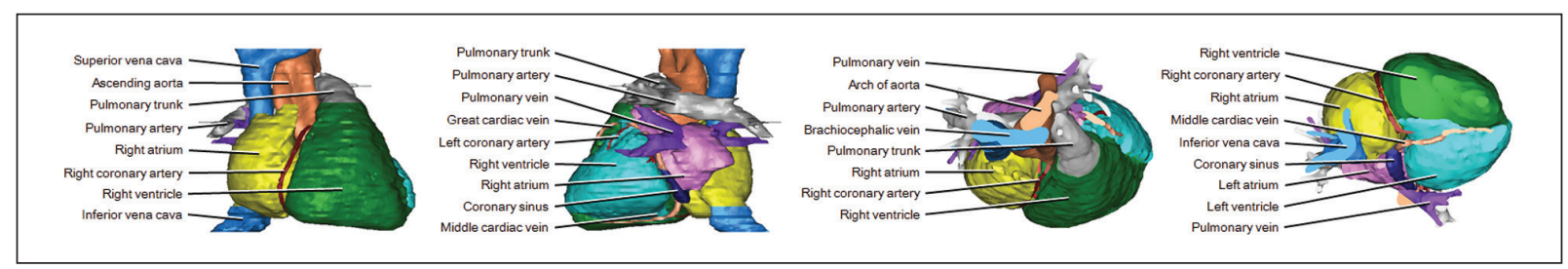

Figure 2. Surface models of the heart components in the anterior, posterior, superior, and inferior views (left to right). Reconstructed structures with specific colours are manually labelled.

images obtained at $5 \mathrm{~mm}$ intervals could be selected to display and rotated by the user (Fig. 3, Table 1).

During exploration of the PDF file, heart variation of this cadaver was detected. The tricuspid valve was located anterior to the mitral valve in the inferior and left views, whereas the tricuspid is known to be right to the mitral [3]. In other words, the heart was rotated clockwise in the inferior prospect. The rotation also resulted in the pulmonary valve that was left to the aortic valve rather than anterior to it. Also, the personal variation was related with the unusual course of the right coronary artery (Fig. 4).

Using advanced software (Acrobat 9.0 Pro Extended; Adobe Systems), the surface models in the PDF file could be sectioned to show the colour-filled images. Consulting to the colours on the planes, labelling on the corresponding sectioned images could be performed without further consideration (Fig. 5). Also, with various quantification functions of the software, size of structures, such as diameter of vessels, could be measured on both 3D models and 2D planes.

\section{DISCUSSION}

Photoshop has been the principle software used for delineation on the sectioned images, due to the capability of the program to manage colour data [5]. This popular software has been preferred by the Visible Korean laboratory [12], the Chinese Visible team [15] and others [2, 14]. The Visible Korean team has carried out the next procedure, surface reconstruction, using assorted software, most recently 3D-DOCTOR version 4 (Able Software, Lexington, MA, USA) [6, 7, 11, 13].

The present study reflects our aim to substitute Mimics as the software for segmentation and reconstruction. There are several reasons for this decision. First, prior to segmentation of structures, their thorough appearance can be grasped. In one MCS fie, researchers are able to scroll through continuous sectioned images comprising the concerned structures. Second, during segmentation, serial outlines of a structure can be simultaneously created. This capacity is more efficient if a long structure is transversely sectioned to be similar in successive 


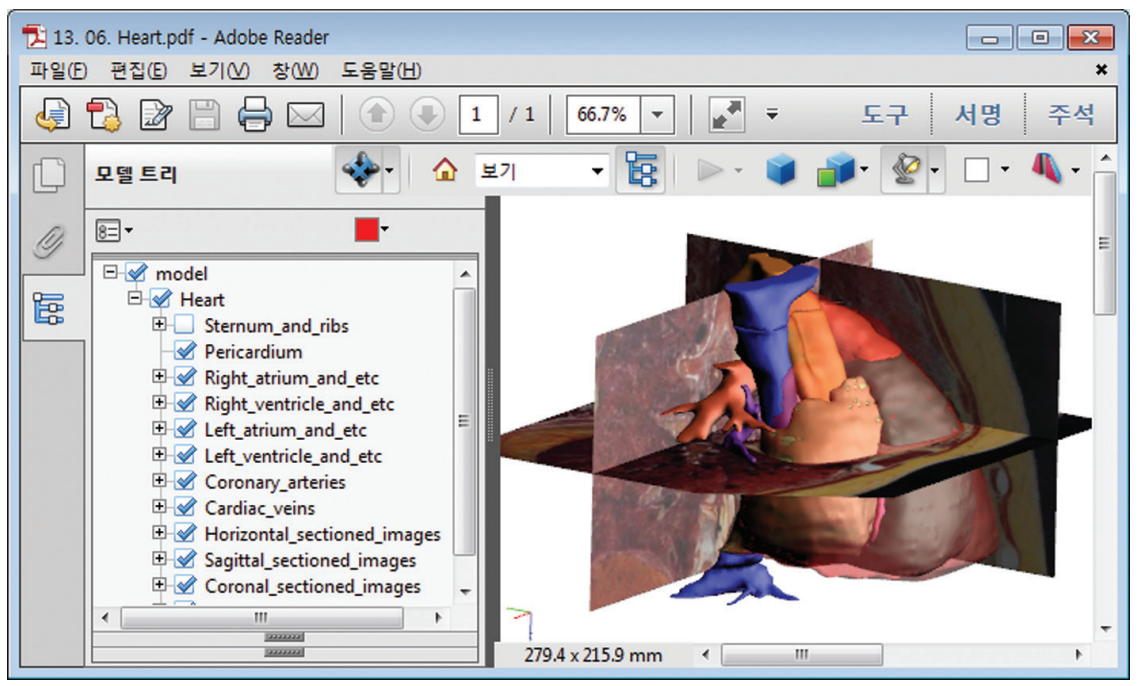

Figure 3. PDF file of the heart, consisting of the left-sided model tree window and the right-sided model display window. The surface models are overlapped by the three sectioned images.

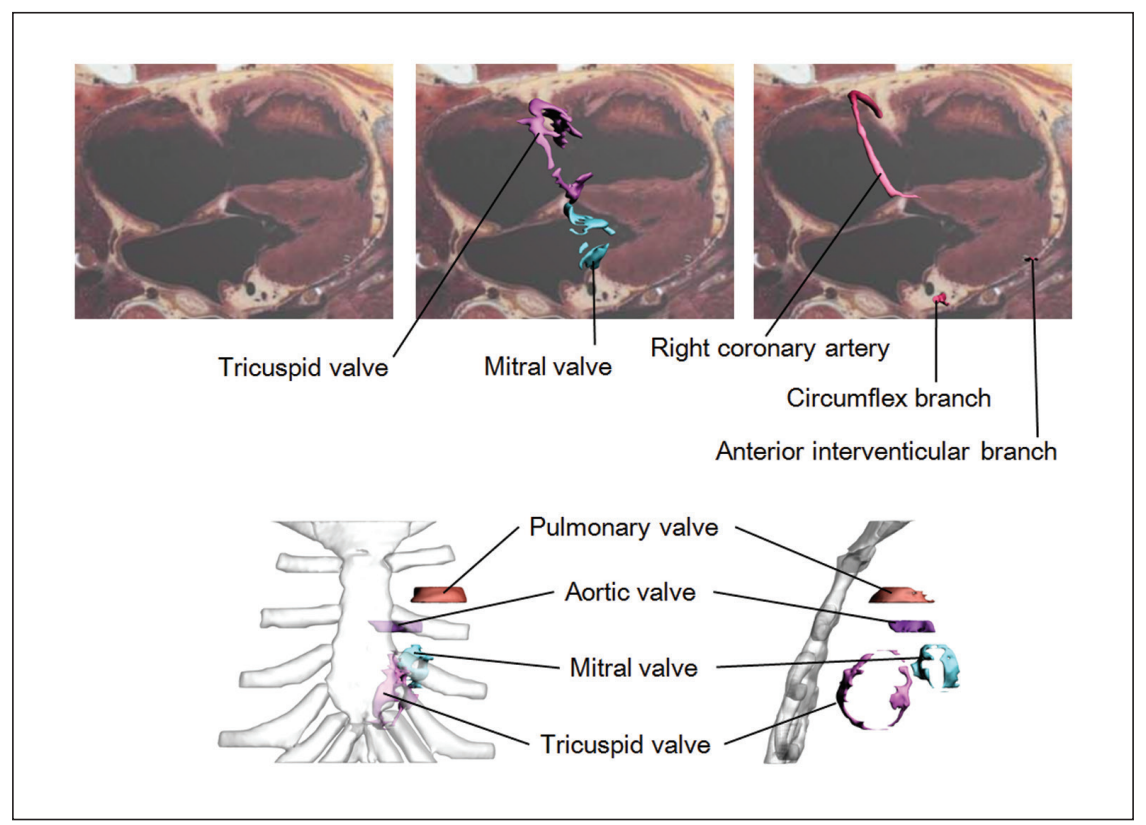

Figure 4. A horizontal sectioned image in the inferior view (top left) that is overlapped either by the surface models of valves (top centre) or by those of coronary arteries (top right). Semitransparent surface models of the bones and valves in the anterior view (bottom left) and in the left view (bottom right).

images. Third, after the segmentation, imperfect contours can be readily searched by scrolling the colour-filled outlines, observing the vertical planes, or referring to the quickly-made surface models (Fig. 1). Furthermore, even if the models are exported from Mimics into other software packages, locational relationships between structures are preserved [1]. On the other hand, 3D-DOCTOR does not keep the positional information.
Nonetheless, Mimics has manifest drawbacks. Firstly, the sectioned images can be seen for delineation; however, their diverse colours automatically become grey like computed tomography (CT) and magnetic resonance images (MRI). Some structures to be outlined are not discernible in the grey level (e.g., muscles with obscure borders). Our suggested solution is to use dual monitors; one of both shows the original colour images. Secondly, Mimics 


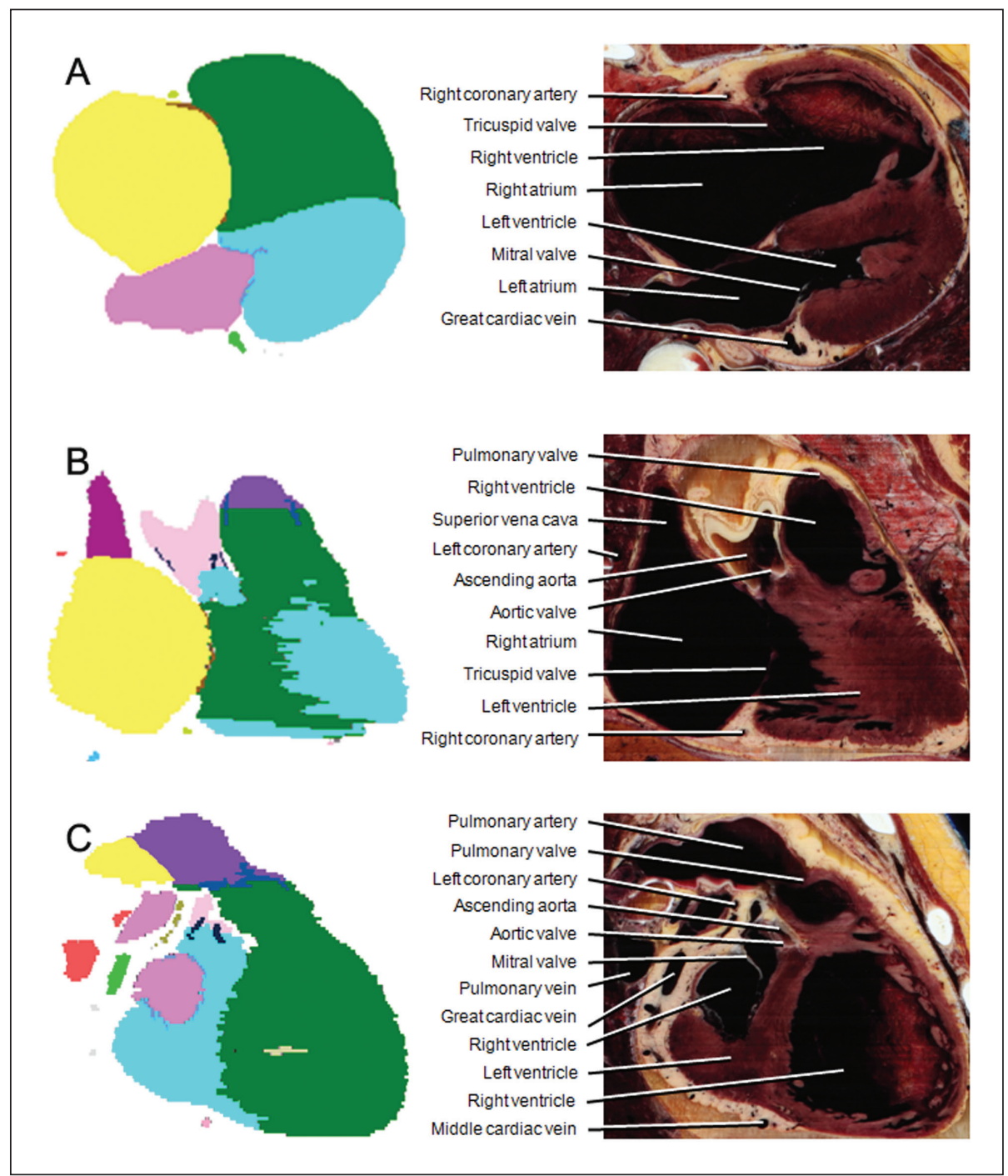

Figure 5. Colour-filled images of heart structures (left column) and corresponding sectioned images, which are annotated (right column) in the horizontal (A), coronal (B), and sagittal (C) planes.

software does not provide diverse drawing options. Unlike Photoshop, only manual illustrating of bitmap style is feasible. A solution is to sketch the outlines in Photoshop, which are then verified and repaired in Mimics, as was done in the present study. Thirdly, due to the memory restriction of Mimics, sufficient images cannot be managed at the same time. One solution is to crop the raw images. Images outside the organs of interest can be cut off. Another solution is to reduce image resolution. Practically, the low quality images are acceptable during demarcation because of the availability of a dual monitor. A third solution is to divide numerous organs into several MCS files. In each file, segmentation and reconstruction of the chosen organs are performed, and the reconstructed models can be easily combined. Compared to the advantages of Mimics, its disadvantages are relatively trivial and can be surmounted. It is anticipated that the next version of Mimics, or competitive software, will eliminate the present deficiencies.

Presently, we generated a PDF file that could be used to interactively visualise the surface models and sectioned images of the heart. The 4 heart chambers are not situated in the simple manner. The right chambers are not only right but also anterior, inferior to the left ones. Likewise, the ventricles are not only inferior but also anterior, left to the atria [3]. The complicated topographic anatomy of heart might be better deduced by a PDF file (Figs. 2, 4, 5). Rotation of the coexisting surface models permits determination of their stereoscopic 
position. For example, the anterior view demonstrates the well-known location of the heart valves on the sternum and ribs, while, after rotation, the resulting lateral view discloses their anteroposterior relationship (Fig. 4).

Combined view of the surface models and corresponding sectioned images is also conducive. In the sectioned images, heart valves and coronary arteries are identifiable, but not simply understandable (Fig. 5). The overlapped surface models are the best choice to explain how the 3D structures look in the 2D planes (Figs. 3, 4). Therefore, our PDF file would be welcomed by medical students, who presently learn about the heart and great vessels using CT, MRI, and ultrasonograph formats.

The 3D models of heart can be extracted from the PDF file by other interested researchers. This is a very unique characteristic of this product, because other software packages do not provide their own raw data due to copyright constraints and the demand for commercial profits. Furthermore, investigators worldwide will be supplied with the full data set of the female cadaver after fulfilling an agreement process with the authors. It is the authors' hope that the complete version could be a robust resource for virtual reality learning of heart and other organs.

\section{CONCLUSIONS}

The Mimics software improves segmentation and surface reconstruction of anatomical constituents in sectioned images. The well-organised PDF file, which is downloadable freely from the Visible Korean homepage, will be a satisfactory self-learning tool of heart anatomy.

\section{ACKNOWLEDGEMENTS}

This research was supported by Basic Science Research Program through the National Research Foundation of Korea (NRF) funded by the Ministry of Education, Science and Technology (grant number 2010-0009950). Raw data of the Visible Korean were acquired by the financial assistance from the Korea Institute of Science and Technology Information.

\section{REFERENCES}

1. Cho SW, Jharia TK, Moon YL, Sim SW, Shin DS, Bigliani LU (2013) Three-dimensional templating arthroplasty of the humeral head. Surg Radiol Anat, 35: 685-688.

2. Lou L, Liu SW, Zhao ZM, Heng PA, Tang YC, Li ZP, Xie YM, Chui YP (2009) Segmentation and reconstruction of hepatic veins and intrahepatic portal vein based on the coronal sectional anatomic dataset. Surg Radiol Anat, 31: 763-768.

3. Moore KL, Dalley AF, Agur AMR (2013) Clinically oriented anatomy. 7th Ed. Lippincott Williams \& Wilkins.

4. Park HS, Chung MS, Shin DS, Jung YW, Park JS (2013) Accessible and informative sectioned images, color-coded images, and surface models of the ear. Anat Rec, 296: 1180-1186.

5. Park JS, Chung MS, Hwang SB, Lee YS, Har DH (2005) Technical report on semiautomatic segmentation using the Adobe Photoshop. J Digit Imaging, 18: 333-343.

6. Park JS, Shin DS, Chung MS, Hwang SB, Chung J (2007) Technique of semiautomatic surface reconstruction of the Visible Korean Human data using commercial software. Clin Anat, 20: 871-879.

7. Shin DS, Chung MS, Park JS (2012) Systematized methods of surface reconstruction from the serial sectioned images of a cadaver head. J Craniofac Surg, 23: 190-194.

8. Shin DS, Chung MS, Park JS, Park HS, Lee S, Moon YL, Jang HG (2012) Portable document format file showing the surface models of cadaver whole body. J Korean Med Sci, 27: 849-856.

9. Shin DS, Jang HG, Hwang SB, Har D-H, Moon YL, Chung MS (2013) Two-dimensional sectioned images and three-dimensional surface models for learning the anatomy of female pelvis. Anat Sci Educ, 6: 316-323.

10. Shin DS, Jang HG, Park JS, Park HS, Lee S, Chung MS (2012) Accessible and informative sectioned images and surface models of a cadaver head. J Craniofac Surg, 23: 1176-1180.

11. Shin DS, Park JS, Chung MS (2012) Three types of the serial segmented images suitable for surface reconstruction. Anat Cell Biol, 45: 128-135.

12. Shin DS, Park JS, Park HS, Hwang SB, Chung MS (2012) Outlining of the detailed structures in sectioned images from Visible Korean. Surg Radiol Anat, 34: 235-247.

13. Shin DS, Park JS, Shin BS, Chung MS (2011) Surface models of the male urogenital organs built from the Visible Korean using popular software. Anat Cell Biol, 44: 151-159.

14. Tang L, Chung MS, Liu Q, Shin DS (2010) Advanced features of whole body sectioned images. Virtual Chinese Human. Clin Anat, 23: 523-529.

15. Wu Y, Tan LW, Li Y, Fang BJ, Xie B, Wu TN, Li QY, Qiu MG, Liu GJ, Li K, Xu HT, Luo N, Zhang SX (2012) Creation of a female and male segmentation dataset based on Chinese Visible Human (CVH). Comput Med Imaging Graph, 36: 336-342. 